\title{
THETA SERIES LIFTINGS FROM ORTHOGONAL GROUPS TO SEMI-SIMPLE GROUPS
}

\author{
MIN HO LEE \\ (Received 3 September 1999; revised 23 February 2000) \\ Communicated by A. H. Dooley
}

\begin{abstract}
We study a correspondence between automorphic forms on an orthogonal group and automorphic forms on a semi-simple Lie group associated to an equivariant holomorphic map of a symmetric domain into a Siegel upper half space. We construct an automorphic form on the symmetric domain that corresponds to an automorphic form on an orthogonal group using theta series, and prove that such a correspondence is compatible with the appropriate Hecke operator actions on the corresponding automorphic forms. As an example, we describe the case of spin groups.
\end{abstract}

2000 Mathematics subject classification: primary $11 \mathrm{~F} 55,11 \mathrm{~F} 27$.

\section{Introduction}

It is well known that a symplectic group and an orthogonal group form a dual reductive pair in the sense of Howe [1], and therefore there is the associated theta correspondence which provides a correspondence between automorphic forms on a symplectic group and automorphic forms on an orthogonal group. The purpose of this paper is to discuss a similar correspondence between automorphic forms on orthogonal groups and automorphic forms on more general semi-simple groups.

In [10] Satake described various aspects of equivariant holomorphic maps of Hermitian symmetric domains. Among such maps we consider the ones associated to symplectic representations of semi-simple Lie groups. Let $\mathbb{G}$ be a semi-simple algebraic group defined over $\mathbb{Q}$, and assume that the Riemannian symmetric space $\mathscr{D}$ associated to the semi-simple Lie group $G=\mathbb{G}(\mathbb{R})$ is a Hermitian symmetric domain. Let $\rho: \mathbb{G} \rightarrow S p_{n}$ be a homomorphism defined over $\mathbb{Q}$, and let $\tau: \mathscr{D} \rightarrow \mathscr{H}_{n}$ be a

(c) 2000 Australian Mathematical Society $0263-6115 / 2000 \$ A 2.00+0.00$ 
holomorphic map of $\mathscr{D}$ into the Siegel upper half space $\mathscr{H}_{n}$ of degree $n$ such that $\rho$ and $\tau$ form an equivariant pair, that is, they satisfy the relation $\tau(g z)=\rho(g) \tau(z)$ for all $z \in \mathscr{H}_{n}$ and $g \in G$. Satake classified such equivariant pairs satisfying some additional conditions. Given such a pair $(\rho, \tau)$ and an arithmetic subgroup $\Gamma$ of $\mathbb{G}(\mathbb{Q})$, we can construct a family of polarized abelian varieties parameterized by the locally symmetric space $\Gamma \backslash \mathscr{D}$, known as the Kuga fiber variety (see for example $[3,4,5,10]$ ).

In this paper we study a correspondence between automorphic forms on an orthogonal group and automorphic forms on the semi-simple group $\mathbb{G}$ associated to an equivariant pair of the above type. More precisely, we construct an automorphic form on the Hermitian symmetric domain $\mathscr{D}$ corresponding to an automorphic form on an orthogonal group using theta series associated to Weil representations. We also prove that such a correspondence is compatible with the appropriate Hecke operator actions on the corresponding automorphic forms. As an example, we describe the case of spin groups associated to Clifford algebras.

\section{Theta series liftings}

Let $\mathbb{G}$ be a semi-simple algebraic group defined over $\mathbb{Q}$ so that its group of real points $G=\mathbb{G}(\mathbb{R})$ is a semi-simple Lie group. Let $K$ be a maximal compact subgroup of $G$, and let $\mathscr{D}=G / K$ be the associated Riemannian symmetric space. We assume that $\mathscr{D}$ has a $G$-invariant complex structure so that it becomes a Hermitian symmetric domain.

DEFINITION 2.1. Let $W$ be a finite-dimensional complex vector space, and let $G L(W)$ be the group of invertible endomorphisms of $W$. If $H$ is a subgroup of $G$, an automorphy factor of $H$ is a map $\mathscr{J}: H \times \mathscr{D} \rightarrow G L(W)$ satisfying the following conditions:

(i) For fixed $g \in H$, the map $z \mapsto \mathscr{J}(g, z), \mathscr{D} \rightarrow G L(W)$ is holomorphic.

(ii) $\mathscr{J}(g h, z)=\mathscr{J}(g, h z) \mathscr{J}(h, z)$ for all $g, h \in H$ and $z \in \mathscr{D}$.

We now modify the usual definition of automorphic forms on the symmetric domain $\mathscr{D}$ by suppressing the growth condition as below.

DEFINITION 2.2. Let $\Gamma$ be a discrete subgroup of $G, \chi: \Gamma \rightarrow \mathbb{C}^{\times}$a character of $\Gamma$, and $\mathscr{J}: \Gamma \times \mathscr{D} \rightarrow G L(W)$ an automorphy factor of $\Gamma$. An automorphic form of type $(\Gamma, \mathscr{J})$ with character $\chi$ is a holomorphic map $f: \mathscr{D} \rightarrow W$ satisfying the functional equation

$$
f(\gamma z)=\chi(\gamma) \mathscr{J}(\gamma, z) f(z)
$$


for all $z \in \mathscr{D}$ and $\gamma \in \Gamma$. We denote by $\mathscr{A}(\Gamma, \mathscr{J}, \chi)$ the space of all automorphic forms of type $(\Gamma, \mathscr{J})$ with character $\chi$.

Given a positive integer $n$, we consider the symplectic group $S p_{n}$ whose rational points are given by

$$
S p_{n}(\mathbb{Q})=\left\{g \in M_{2 n, 2 n}(\mathbb{Q}) \mid g J_{n}{ }^{t} g=J_{n}\right\}, \quad J_{n}=\left(\begin{array}{cc}
0 & I_{n} \\
-I_{n} & 0
\end{array}\right),
$$

where $M_{2 n, 2 n}(\mathbb{Q})$ is the set of $2 n \times 2 n$ matrices with entries in $\mathbb{Q}$ and $I_{n}$ is the $n \times n$ identity matrix. The Hermitian symmetric domain associated to the simple Lie group $S p_{n}(\mathbb{R})$ is identified as usual with the Siegel upper half space

$$
\mathscr{H}_{n}=\left\{\left.Z \in M_{n, n}(\mathbb{C})\right|^{t} Z=Z, \quad \operatorname{Im} Z \gg 0\right\}
$$

of degree $n$, so that the action of $S p_{n}(\mathbb{R})$ on $\mathscr{H}_{n}$ is given by

$$
g Z=(A Z+B)(C Z+D)^{-1}, \quad g=\left(\begin{array}{ll}
A & B \\
C & D
\end{array}\right) \in S p_{n}(\mathbb{R}), \quad Z \in \mathscr{H}_{n} .
$$

Let $K_{\infty}^{\prime}$ be the maximal compact subgroup of $S p_{n}(\mathbb{R})$ consisting of the elements that fix the point $i I_{n} \in \mathscr{H}_{n}$. We assume that there exist a homomorphism $\rho: \mathbb{G} \rightarrow S p_{n}$ defined over $\mathbb{Q}$ and a holomorphic map $\tau: \mathscr{D} \rightarrow \mathscr{H}_{n}$ such that $\tau(g z)=\rho(g) \tau(z)$ for all $g \in G=\mathbb{G}(\mathbb{R})$ and $z \in \mathscr{D}$. In particular, we have $\rho(K) \subset K^{\prime}$.

REMARK 2.3. Various aspects of equivariant pairs $(\rho, \tau)$ of the above type as well as more general equivariant pairs were described extensively by Satake in [10]. In particular, such an equivariant pair and a discrete subgroup $\Gamma$ of $G$ determine a Kuga fiber variety, which is a family of polarized abelian varieties parameterized by the locally symmetric variety $\Gamma \backslash \mathscr{D}$ (see $[3,4,5,10]$ ).

Let $V$ be a vector space of even dimension $m$ over $\mathbb{Q}$ equipped with a positive definite quadratic form $\mathscr{Q}(x)$. Let $L$ be a lattice in $V$ of the form $L=\mathbb{Z} v_{1}+\cdots+\mathbb{Z} v_{m}$ for some basis $\left\{v_{i}\right\}$ of $V$. Using this basis, we can identify each element $x \in V$ with a vector in $\mathbb{Q}^{n}$, and under this identification we have

$$
\mathscr{Q}(x)=Q[x]={ }^{t} x Q x
$$

for some positive definite $m \times m$ matrix $Q$. Note that the quadratic form $\mathscr{Q}(x)$ determines a bilinear form $B(\cdot, \cdot)$ on $V$ given by

$$
B(x, y)=\mathscr{Q}(x+y)-\mathscr{Q}(x)-\mathscr{Q}(y)
$$


for all $x, y \in V$, which satisfies the relations

$$
B(x, y)={ }^{t} x(2 Q) y, \quad \mathscr{Q}(x)=(1 / 2) B(x, x) .
$$

We assume that $L$ is an integral lattice, that is, $\mathscr{Q}(x) \in \mathbb{Z}$ for all $x \in L$. Then $\mathscr{Q}(x)$ determines a quadratic form on $V_{p}=V \otimes_{\mathbf{Q}} \mathbb{Q}_{p}$ for each prime $p$, and the matrix $2 Q$ has integral entries with even numbers on the diagonal. We denote by $O_{\mathbf{Q}}=O_{\mathbf{Q}}(\mathscr{Q})$ $\left(O_{\infty}=O_{\mathbb{R}}(\mathscr{Q})\right.$ ) the orthogonal group of the quadratic form $\mathscr{Q}(x)$ over $\mathbb{Q}(\mathbb{R})$. If we set

$$
L_{p}^{\prime}=\left\{x \in V_{p} \mid B(x, y) \in \mathbb{Z}_{p} \text { for all } y \in L_{p}\right\},
$$

then for each prime $p$ there is a nonnegative integer $j_{p}$ such that

$$
\mathbb{Z}_{p}\left\langle\mathscr{Q}(x) \mid x \in L_{p}^{\prime}\right\rangle=p^{j_{p}} \mathbb{Z}_{p} .
$$

Then the integer $q$ defined by

$$
q=\prod_{p} p^{j_{p}}
$$

is called the level of the integral lattice $L$.

Let $\left(V_{\lambda}, \lambda\right)$ and $\left(V_{\mu}, \mu\right)$ be irreducible unitary representations of $O_{\infty}$ and $K_{\infty}^{\prime}$, respectively. Using the basis determined by the lattice $L$, we can identify the space $V_{\mathbb{R}}^{n}=V^{n} \otimes_{\mathbb{Q}} \mathbb{R}$ with the space $M_{m, n}(\mathbb{R})$ of $m \times n$ real matrices. We assume that there is a polynomial map

$$
P: M_{m, n}(\mathbb{R}) \rightarrow \operatorname{Hom}_{C}\left(V_{\lambda}, V_{\mu}\right)
$$

satisfying the conditions

$$
P\left(A^{-1} X\right)=P(X) \lambda(A), \quad P(X B)=\mu\left({ }^{\prime} B\right) P(X)
$$

for all $A \in O_{\mathbb{R}}(\mathscr{Q})$ and $B \in G L_{n}(\mathbb{C})$; here we used the natural extension of $\mu$ to a representation of $G L_{n}(\mathbb{C})$ as described in [2, Section 3.2].

Let $\mathbb{A}$ be the ring of adeles of $\mathbb{Q}$, and let $O_{\mathrm{A}}=O_{\mathrm{A}}(\mathscr{Q})$ be the orthogonal group of the quadratic form $\mathscr{Q}(x)$ over $\mathbb{A}$. For each place $v$ of $\mathbb{Q}$, we define the subgroup $U_{v}$ of $O_{v}=O_{\mathbf{Q}_{v}}(\mathscr{Q})$ by $U_{\infty}=O_{\infty}=O_{\mathbb{R}}$ and

$$
U_{p}=\left\{g \in O_{p} \mid g L_{p}=L_{p}\right\}
$$

for $p<\infty$. Then $U=\prod_{v} U_{v} \subset O_{\mathrm{A}}$ becomes the stabilizer of $L$ for the action of the group $O_{\AA}$ on $V_{\mathrm{A}}$, and $O_{\mathrm{A}}$ has a decomposition of the form

$$
o_{\mathrm{A}}=\bigcup_{i=1}^{\nu} o_{\mathbf{Q}} \alpha_{i} U
$$


for some $\alpha_{1}, \ldots, \alpha_{n} \in O_{\mathrm{A}}$. We extend the representation $\left(V_{\lambda}, \lambda\right)$ of $O_{\infty}$ to a representation of $U$ by setting $\lambda(u)=\lambda\left(u_{\infty}\right)$ for $u=\left(u_{v}\right) \in U$, and define automorphic forms on orthogonal groups without the growth conditions as follows:

DEFINITION 2.4. An automorphic form on $O_{\mathrm{A}}$ of type $(U, \lambda)$ is a function $f$ : $O_{\mathrm{A}} \rightarrow V_{\lambda}$ satisfying the relation

$$
f(a x u)=f(x) \lambda(u)
$$

for all $a \in O_{\mathbf{Q}}, x \in O_{\mathrm{A}}$ and $u \in U$. We denote by $\mathscr{A}_{\lambda}\left(O_{\mathbf{Q}} \backslash O_{\mathrm{A}} / U\right)$ the space of all automorphic forms on $O_{A}$ of type $(U, \lambda)$.

For each place $v$ of $\mathbb{Q}$, let $V_{v}=V \otimes_{\mathbb{Q}} \mathbb{Q}_{v}$, and let $\mathscr{S}\left(V_{v}^{n}\right)$ be the space of SchwartzBruhat functions on $V_{v}^{n}$. We denote by $\pi_{v}$ the local Weil representation of $S p_{n}\left(\mathbb{Q}_{v}\right)$ on $\mathscr{S}\left(V_{v}^{n}\right)$ (see [11, Section 3]). Let $\mathscr{S}\left(V_{\AA}^{n}\right)$ be the space of functions on $V_{v}^{n}$ of the form $\psi=\prod_{v} \psi_{v}$ with $\psi_{v} \in \mathscr{S}\left(V_{v}^{n}\right)$ for each place $v$ such that $\psi_{p}$ is the characteristic function of $L_{p}^{n}$ for all but a finite number of finite primes $p$. Then the global Weil representation $\pi$ of $S p_{n}(\mathbb{A})$ on $\mathscr{S}\left(V_{\mathbb{A}}^{n}\right)$ is determined by using the formula

$$
\pi(h) \psi=\prod_{v} \pi_{v}\left(h_{v}\right) \psi_{v}, \quad h=\left(h_{v}\right) \in S p_{n}(\mathbb{A})
$$

and extending it to the whole space $\mathscr{S}\left(V_{\AA}^{n}\right)$ by continuity.

In order to consider the Weil representation of $O_{\mathrm{A}}$ we first define the representation $\pi_{v}^{\prime}$ of $O_{v}$ on $\mathscr{S}\left(V_{v}^{n}\right)$ by

$$
\pi_{v}^{\prime}\left(g_{v}\right) \psi_{v}(x)=\psi_{v}\left(g_{v}^{-1} x\right)
$$

for $g_{v} \in O_{v}, \psi_{v} \in \mathscr{S}\left(V_{v}^{n}\right)$ and $x \in V_{v}^{n}$. Then the global Weil representation $\pi^{\prime}$ of $O_{\mathrm{A}}$ on $\mathscr{S}\left(V_{\mathrm{A}}^{n}\right)$ is given by

$$
\pi^{\prime}(g) \psi=\prod_{v} \pi_{v}^{\prime}\left(g_{v}\right) \psi_{v}
$$

for $g=\left(g_{v}\right) \in O_{\mathbb{A}}$ and $\psi=\prod_{v} \psi_{v} \in \mathscr{S}\left(V_{\AA}^{n}\right)$.

Given an element $\psi \in \mathscr{S}\left(V_{\AA}^{n}\right)$, we set

$$
\tilde{\psi}_{\rho}\left(g, g^{\prime}\right)=\sum_{X \in V^{n}} \pi(\rho(g)) \pi^{\prime}\left(g^{\prime}\right) \psi(X)
$$

for $g \in \mathbb{G}(\mathbb{A})$ and $g^{\prime} \in O_{\mathbb{A}}$, where $\rho: \mathbb{G}(\mathbb{A}) \rightarrow S p_{n}(\mathbb{A})$ is the map determined by $\rho: \mathbb{G} \rightarrow S p_{n}$ that is equivariant with the holomorphic map $\tau: \mathscr{D} \rightarrow \mathscr{H}_{n}$ described above. Since the function

$$
\tilde{\psi}\left(h, g^{\prime}\right)=\sum_{X \in V^{n}} \pi(h) \pi^{\prime}\left(g^{\prime}\right) \psi(X)
$$


for $h \in S p_{n}(\mathbb{A})$ and $g^{\prime} \in O_{\mathbf{A}}$ is known to be left $S p_{n}(\mathbb{Q})$-invariant and right $O_{\mathbf{Q}^{-}}$ invariant (see [11, Section 4.3]), we have

$$
\widetilde{\psi}_{\rho}\left(\gamma g, \gamma^{\prime} g^{\prime}\right)=\tilde{\psi}_{\rho}\left(g, g^{\prime}\right)
$$

for all $\gamma \in \mathbb{G}(\mathbb{Q})$ and $\gamma^{\prime} \in O_{\mathbf{Q}}$. We assume that there exists an element $z_{0} \in \mathscr{D}$ such that $\tau\left(z_{0}\right)=i I_{n}$, and for $\psi \in \mathscr{S}\left(V_{\mathrm{A}}^{n}\right)$ and $f \in \mathscr{A}_{\lambda}\left(O_{\mathbf{Q}} \backslash O_{\mathrm{A}} / U\right)$ we set

$$
\Theta(\psi, f)(z)=\left(\operatorname{det}^{m / 2} \otimes \mu\right)\left(J\left(\rho\left(g_{\infty}\right), i I_{n}\right)\right) \int_{O_{\mathbf{Q}} \backslash O_{A}}\left\langle\tilde{\psi}_{\rho}\left(g, g^{\prime}\right), f\left(g^{\prime}\right)\right) d g^{\prime},
$$

where $z=g_{\infty} z_{0} \in \mathscr{D}, g=\left(g_{\infty}, 1,1, \ldots\right) \in \mathbb{G}(\mathbb{A})$, and $J: S p_{n}(\mathbb{R}) \times \mathscr{H}_{n} \rightarrow \mathbb{C}$ is the automorphy factor of $S p_{n}(\mathbb{R})$ given by

$$
J\left(g^{\prime}, Z\right)=C Z+D, \quad g^{\prime}=\left(\begin{array}{ll}
A & B \\
C & D
\end{array}\right) \in S p_{n}(\mathbb{R}), \quad Z \in \mathscr{H}_{n} .
$$

We also set

$$
\Gamma_{0}^{n}(q)=\left\{\left(\begin{array}{ll}
A & B \\
C & D
\end{array}\right) \in S p_{n}(\mathbb{Z}) \mid C \equiv 0 \quad(\bmod q)\right\},
$$

where $q$ is the level of the lattice $L$, and consider a discrete subgroup $\Gamma$ of $G$ such that $\rho(\Gamma) \subset \Gamma_{0}^{n}(q)$. Then it can be easily seen that the function $\mathscr{J}_{\rho, \tau}^{\mu}: \Gamma \times \mathscr{D} \rightarrow G L\left(V_{\mu}\right)$ given by

$$
\mathscr{J}_{\rho, \tau}^{\mu}(\gamma, z)=\left(\operatorname{det}^{m / 2} \otimes \mu\right)(J(\rho(\gamma), \tau(z)))
$$

is an automorphy factor of $\Gamma$.

REMARK 2.5. The automorphy factor $\mathscr{J}_{\rho, \tau}^{\mu}$ above may be regarded as a special case of automorphy factors for mixed automorphic forms studied in [7] (see also [6]) which are linked to the geometry of the family of abelian varieties parameterized by a locally symmetric variety associated to the equivariant pair $(\rho, \tau)$ described above.

Let $d=(-1)^{m / 2} \operatorname{det}(2 Q)$ be the discriminant of the quadratic form $\mathscr{Q}$, and let $(\cdot, \cdot)_{p}$ denote the Hilbert symbol for $\mathbb{Q}_{p}$ for each finite prime $p$. Given a place $v$ of $\mathbb{Q}$ we define the character $\chi_{v}$ of $\mathbb{Q}_{v}^{\times}$by $\chi_{v}(x)=(x, D)_{v}$ if $v$ is a finite prime $p$, $\chi_{\infty}(x)=1$ for $d>0$ and $\chi_{\infty}(x)=\operatorname{sgn} x$ for $d<0$. Let $\chi$ be the character of $\Gamma_{0}^{n}(q)$ given by

$$
\chi(\gamma)=\prod_{p \mid q} \chi_{p}(\operatorname{det} A) \text { for } \gamma=\left(\begin{array}{ll}
A & B \\
C & D
\end{array}\right) \in \Gamma_{0}^{n}(q) .
$$

Since $\rho(\Gamma) \subset \Gamma_{0}^{n}(q)$, we see that $\chi \circ \rho$ is a character of $\Gamma$. We now state the theorem that provides a correspondence between automorphic forms on $O_{\mathrm{A}}$ and automorphic forms on $\mathscr{D}$ with character $\chi \circ \rho$. 
THEOREM 2.6. Let $\mathscr{J}_{\rho, \tau}^{\mu}$ be the automorphy factor described above, and let $f$ be an automorphic form in $\mathscr{A}_{\lambda}\left(O_{\mathbf{Q}} \backslash O_{\mathrm{A}} / U\right)$. If the discrete subgroup $\Gamma$ of $G$ and the character $\chi$ of $\Gamma_{0}^{n}(q)$ are as above, then the function $\Theta(\psi, f): \mathscr{D} \rightarrow V_{\mu}$ is an automorphic form in $\mathscr{A}\left(\Gamma, \mathscr{J}_{\rho, \tau}^{\mu}, \chi \circ \rho\right)$ of type $\left(\Gamma, \mathscr{J}_{\rho, \tau}^{\mu}\right)$ with character $\chi \circ \rho$.

PROOF. Let $\widetilde{\psi}\left(h, g^{\prime}\right)=\sum_{X \in V^{n}} \pi(h) \pi^{\prime}\left(g^{\prime}\right) \psi(X)$ for $h \in S p_{n}(\mathbb{A})$ and $g^{\prime} \in O_{\mathbb{A}}$ be as before, and set

$$
\Theta^{\prime}(\psi, f)\left(z^{\prime}\right)=\left(\operatorname{det}^{m / 2} \otimes \mu\right)\left(J\left(h_{\infty}, i I_{n}\right)\right) \int_{O_{Q} \backslash O_{\wedge}}\left\langle\tilde{\psi}_{\rho}\left(h, g^{\prime}\right), f\left(g^{\prime}\right)\right\rangle d g^{\prime}
$$

for $z^{\prime}=h_{\infty} z_{0} \in \mathscr{H}_{n}$. Then, since $\tau(z)=\tau\left(g_{\infty} z_{0}\right)=\rho\left(g_{\infty}\right)\left(i I_{n}\right)$, we have

$$
\Theta(\psi, f)(z)=\Theta^{\prime}(\psi, f)(\tau(z))
$$

for all $z \in \mathscr{D}$. However, using [11, Proposition 5.1], we obtain

$$
\begin{aligned}
\Theta^{\prime}(\psi, f)(\tau(\gamma z)) & =\Theta^{\prime}(\psi, f)(\rho(\gamma) \tau(z)) \\
& =\chi(\gamma)\left(\operatorname{det}^{m / 2} \otimes \mu\right)(J(\rho(\gamma), \tau(z))) \Theta^{\prime}(\psi, f)(\tau(z)),
\end{aligned}
$$

which implies that

$$
\Theta(\psi, f)(\gamma z)=\chi(\gamma)\left(\operatorname{det}^{m / 2} \otimes \mu\right)(J(\rho(\gamma), \tau(z))) \Theta(\psi, f)(z),
$$

and therefore the theorem follows.

\section{Hecke operators}

In this section we describe Hecke operators acting on the spaces $\mathscr{A}\left(\Gamma, \mathscr{J}_{\rho, \tau}^{\mu}, \chi \circ \rho\right)$ and $\mathscr{A}_{\lambda}\left(O_{\mathrm{Q}} \backslash O_{\mathrm{A}} / U\right)$, and show that the Hecke operator action on $\Theta(\psi, f) \in \mathscr{A}(\Gamma$, $\left.\mathscr{J}_{\rho, \tau}^{\mu}, \chi \circ \rho\right)$ is compatible with the Hecke operator action on $f \in \mathscr{A}_{\lambda}\left(O_{\mathbb{Q}} \backslash O_{\mathbb{A}} / U\right)$. Given a prime $p$ that does not divide the level $q$ of $L$, we set

$$
\Delta_{0}^{n}(q)=\left\{\left(\begin{array}{ll}
A & B \\
C & D
\end{array}\right) \in S p_{n}\left(\mathbb{Z}\left[p^{-1}\right]\right) \mid C \equiv 0 \quad(\bmod q)\right\},
$$

where $\mathbb{Z}\left[p^{-1}\right]=\left\{a / p^{b} \mid a, b \in \mathbb{Z}, b \geq 0\right\}$. Let $\Gamma$ be a discrete subgroup of $G$ with $\rho(\Gamma) \subset \Gamma_{0}^{n}(q)$ as in Section 2, and let $\Delta$ be another subgroup of $G$ containing $\Gamma$ such that $\rho(\Delta) \subset \Delta_{0}^{n}(q)$. Given an element $\gamma \in \Delta$ and a function $\theta: \mathscr{D} \rightarrow V_{\mu}$ we set

$$
(\theta \mid \gamma)(z)=\chi(\rho(\gamma))\left(\operatorname{det}^{m / 2} \otimes \mu\right) J(\rho(\gamma), \tau(z))^{-1} \theta(\gamma z)
$$


for all $z \in \mathscr{D}$. Let $H(\Gamma, \Delta)$ be the Hecke ring determined by the pair $(\Gamma, \Delta)$. If $T \in H(\Gamma, \Delta)$ has a decomposition of the form

$$
T=\sum_{i=1}^{\nu} c_{i}\left(\Gamma \alpha_{i}\right), \quad \alpha_{1}, \ldots, \alpha_{\nu} \in \Delta,
$$

we set

$$
\theta \mid T=\sum_{i=1}^{\nu} c_{i}\left(\theta \mid \alpha_{i}\right) .
$$

This determines an action of the Hecke ring $H(\Gamma, \Delta)$ on the space $\mathscr{A}\left(\Gamma, \mathscr{J}_{\rho, \tau}^{\mu}, \chi \circ \rho\right)$ of automorphic forms of type $\left(\Gamma, \mathscr{J}_{\rho, \tau}^{\mu}\right)$ with character $\chi \circ \rho$.

Now we want to consider Hecke operators on the space $\mathscr{A}_{\lambda}\left(O_{\mathbf{Q}} \backslash O_{\mathrm{A}} / U\right)$ of automorphic forms on $O_{\mathrm{A}}$ of type $(U, \lambda)$. For a finite prime $p$ let $H\left(U_{p}, O_{p}\right)$ be the Hecke ring of the pair $\left(U_{p}, O_{p}\right)$, where $U_{p}$ is the stabilizer of $L_{p}$ in $O_{p}$. If $T^{\prime} \in H\left(U_{p}, O_{p}\right)$ has a decomposition of the form

$$
T^{\prime}=\sum_{i=1}^{v^{\prime}} c_{i}^{\prime}\left(U_{p} \alpha_{i}^{\prime}\right), \quad \alpha_{1}^{\prime}, \ldots, \alpha_{v^{\prime}}^{\prime} \in O_{p},
$$

we set

$$
\left(f \mid T^{\prime}\right)(g)=\sum_{i=1}^{\nu^{2}} c_{i}^{\prime} f\left(g \alpha^{\prime-1}\right)
$$

for $f \in \mathscr{A}_{\lambda}\left(O_{\mathrm{Q}} \backslash O_{\mathbb{A}} / U\right)$ and $g \in O_{\mathrm{A}}$. If $\Delta \supset \Gamma$ is a subgroup of $G$ with $\rho(\Delta) \subset \Gamma_{0}^{n}(q)$ as above and if $\delta \in \Delta$, then we denote by $\widetilde{\delta}$ the element of $G(A)$ given by

$$
\widetilde{\delta}=(1, \delta, \delta, \ldots),
$$

and set

$$
\psi^{\delta}=\psi_{\infty}\left[\pi_{p}\left(\rho\left(\delta^{-1}\right) \psi_{p}\right)\right] \prod_{p^{\prime} \neq p} \psi_{p^{\prime}}
$$

for $\psi \in \mathscr{S}\left(V_{A}^{n}\right)$

LEMMA 3.1. Given an automorphic form $f \in \mathscr{A}_{\lambda}\left(O_{\mathbf{Q}} \backslash O_{\mathbf{A}} / U\right)$ and an element $\psi \in \mathscr{S}\left(V_{\mathrm{A}}^{n}\right)$, we have

$$
(\Theta(\psi, f) \mid \delta)=\Theta\left(\psi^{\delta}, f\right)
$$

for all $\delta \in \Delta$.

PROOF. For each $z \in \mathscr{D}$ we have

$(\Theta(\psi, f) \mid \delta)(z)=\chi\left((\rho(\delta))\left(\operatorname{det}^{m / 2} \otimes \mu\right)\left(J(\rho(\delta), \tau(z))^{-1}\right) \Theta(\psi, f)(\delta z)\right.$ 


$$
\begin{aligned}
= & x\left((\rho(\delta))\left(\operatorname{det}^{m / 2} \otimes \mu\right)\left(J(\rho(\delta), \tau(z))^{-1}\right)\right. \\
& \times\left(\operatorname{det}^{m / 2} \otimes \mu\right)\left(J\left(\rho\left(\delta g_{\infty}\right), i I_{n}\right)\right) \int_{O_{\mathbf{Q}} \backslash O_{A}}\left\langle\tilde{\psi}_{\rho}\left(\delta g, g^{\prime}\right), f\left(g^{\prime}\right)\right\rangle d g^{\prime},
\end{aligned}
$$

where $z=g_{\infty} z_{0}$ and $\delta g=\left(\delta g_{\infty}, 1,1, \ldots\right) \in \mathbb{G}(\mathbb{A})$. Since

$$
\rho\left(g_{\infty}\right) i I_{n}=\rho\left(g_{\infty}\right) \tau\left(z_{0}\right)=\tau\left(g_{\infty} z_{0}\right)=\tau(z),
$$

we see that

$$
\begin{aligned}
J\left(\rho\left(\delta g_{\infty}\right), i I_{n}\right) & =J\left(\rho(\delta), \rho\left(g_{\infty}\right) i I_{n}\right) J\left(\rho\left(g_{\infty}\right), i I_{n}\right) \\
& =J(\rho(\delta), \tau(z)) J\left(\rho\left(g_{\infty}\right), i I_{n}\right) .
\end{aligned}
$$

On the other hand, we also have

$$
\delta g=\left(\delta g_{\infty}, 1,1, \ldots\right)=(\delta, \delta, \ldots) g\left(1, \delta^{-1}, \delta^{-1}, \ldots\right)=(\delta, \delta, \ldots) g \widetilde{\delta}^{-1} .
$$

Hence, using the fact that $\widetilde{\psi}_{\rho}$ is left $\mathbb{G}(\mathbb{Q})$-invariant, we obtain

$$
\begin{aligned}
(\Theta(\psi, f) \mid \delta)(z)= & \chi\left((\rho(\delta))\left(\operatorname{det}^{m / 2} \otimes \mu\right)\left(J\left(\rho\left(g_{\infty}\right), i I_{n}\right)\right)\right. \\
& \times \int_{O_{\mathbf{Q} \backslash O_{A}}}\left\langle\sum_{X \in V^{n}} \pi\left(\rho\left(g \tilde{\delta}^{-1}\right)\right) \pi^{\prime}\left(g^{\prime}\right) \psi(X), f\left(g^{\prime}\right)\right\rangle d g^{\prime} .
\end{aligned}
$$

Since the representations $\pi$ and $\pi^{\prime}$ commute (see [11, Section 4.2]), we have

$$
\sum_{X \in V^{n}} \pi\left(\rho\left(g \tilde{\delta}^{-1}\right)\right) \pi^{\prime}\left(g^{\prime}\right) \psi(X)=\sum_{X \in V^{n}} \pi(\rho(g)) \pi^{\prime}\left(g^{\prime}\right)\left[\pi\left(\rho\left(\tilde{\delta}^{-1}\right)\right) \psi\right](X) .
$$

Now, using the relation

$$
\begin{aligned}
\chi\left((\rho(\delta)) \pi\left(\rho\left(\tilde{\delta}^{-1}\right)\right) \psi\right. & =\chi\left((\rho(\delta)) \psi_{\infty}\left[\pi_{p}\left(\rho\left(\tilde{\delta}^{-1}\right)\right) \psi_{p}\right] \prod_{p^{\prime} \neq p} \pi_{p^{\prime}}\left(\rho\left(\tilde{\delta}^{-1}\right)\right) \psi_{p^{\prime}}\right. \\
& =\psi_{\infty}\left[\pi_{p}\left(\rho\left(\delta^{-1}\right) \psi_{p}\right)\right] \prod_{p^{\prime} \neq p} \psi_{p^{\prime}}=\psi^{\delta},
\end{aligned}
$$

we obtain

$$
\begin{aligned}
(\Theta(\psi, f) \mid \delta)(z) & =\left(\operatorname{det}^{m / 2} \otimes \mu\right)\left(J\left(\rho\left(g_{\infty}\right), i I_{n}\right)\right) \int_{O_{Q} \backslash O_{\Lambda}}\left\langle\widetilde{\psi}_{\rho}^{\delta}\left(g, g^{\prime}\right), f\left(g^{\prime}\right)\right\rangle d g^{\prime} \\
& =\Theta\left(\psi^{\delta}, f\right)(z),
\end{aligned}
$$

and hence the proof of the lemma is complete. 
For a finite prime $p$ we set

$$
\Gamma_{p}^{n}(q)=\left\{\left(\begin{array}{ll}
A & B \\
C & D
\end{array}\right) \in S p_{n}\left(\mathbb{Z}_{p}\right) \mid C \equiv 0 \quad(\bmod q)\right\} .
$$

Then $\Gamma_{p}^{n}(q)$ is a compact subgroup of $S p_{n}\left(\mathbb{Q}_{p}\right)$ and is equal to $S p_{n}\left(\mathbb{Z}_{p}\right)$ if $p$ is prime to $q$. If $H\left(\Gamma_{p}^{n}(q), \Delta_{p}^{n}(q)\right)$ is the Hecke ring for the pair $\left(\Gamma_{p}^{n}(q), \Delta_{p}^{n}(q)\right)$, then there is an isomorphism

$$
\varepsilon: H\left(\Gamma_{0}^{n}(q), \Delta_{0}^{n}(q)\right) \rightarrow H\left(\Gamma_{p}^{n}(q), \Delta_{p}^{n}(q)\right)
$$

given by

$$
\varepsilon\left(\sum_{i=1}^{v^{0}} c_{i}^{0}\left(\Gamma_{0}^{n}(q) \alpha_{i}^{0}\right)\right)=\sum_{i=1}^{\nu^{0}} c_{i}^{0}\left(\Gamma_{p}^{n}(q) \alpha_{i}^{0}\right) .
$$

We also note that the anti-automorphism $\iota$ of $H\left(\Gamma_{p}^{n}(q), \Delta_{p}^{n}(q)\right)$ given by

$$
\iota\left(\sum_{i=1}^{v^{1}} c_{i}^{1}\left(\Gamma_{p}^{n}(q) \alpha_{i}^{1}\right)\right)=\sum_{i=1}^{v^{1}} c_{i}^{1}\left(\left(\alpha_{i}^{1}\right)^{-1} \Gamma_{p}^{n}(q)\right)
$$

is actually the identity map, and define the action of the Hecke ring $H\left(\Gamma_{p}^{n}(q), \Delta_{p}^{n}(q)\right)$ on the function $\psi_{p} \in \mathscr{S}\left(V_{p}^{n}\right)$ by

$$
\pi_{p}\left(T_{p}^{1}\right) \psi_{p}=\sum_{i=1}^{\nu^{1}} c_{i}^{1} \pi_{p}\left(\alpha_{i}^{1}\right) \psi_{p}
$$

for

$$
T_{p}^{1}=\sum_{i=1}^{\nu^{1}} c_{i}^{1}\left(\alpha_{i}^{1} \Gamma_{p}^{n}(q)\right) \in H\left(\Gamma_{p}^{n}(q), \Delta_{p}^{n}(q)\right), \quad \alpha_{1}^{1}, \ldots, \alpha_{\nu^{1}}^{1} \in \Delta_{p}^{n}(q) .
$$

We further note that $\rho: \mathbb{G} \rightarrow S p_{n}$ induces a ring homomorphism

$$
\tilde{\rho}: H(\Gamma, \Delta) \rightarrow H\left(\Gamma_{0}^{n}(q), \Delta_{0}^{n}(q)\right)
$$

given by

$$
\tilde{\rho}\left(\sum_{i=1}^{\nu} c_{i}\left(\Gamma \alpha_{i}\right)\right)=\sum_{i=1}^{\nu} c_{i}\left(\Gamma_{0}^{n}(q) \rho\left(\alpha_{i}\right)\right) .
$$

PROPOSITION 3.2. For each $T \in H(\Gamma, \Delta)$ we have

$$
(\Theta(\psi, f) \mid T)(z)=\Theta\left(\psi^{0}, f\right)(z)
$$

for all $z \in \mathscr{D}, \psi \in \mathscr{S}\left(V_{\mathrm{A}}^{n}\right)$ and $f \in \mathscr{A}_{\lambda}\left(O_{\mathbf{Q}} \backslash O_{\mathrm{A}} / U\right)$, where

$$
\psi^{0}=\prod_{\nu} \psi_{v}^{0}, \quad \psi_{v}^{0}= \begin{cases}\psi_{v} & \text { for } v \neq p ; \\ \pi_{p}(\varepsilon \circ \tilde{\rho}(T)) \psi_{p} & \text { for } v=p .\end{cases}
$$


PROOF. Let $T=\sum_{i=1}^{v} c_{i}\left(\Gamma \alpha_{i}\right)$ with $\alpha_{1}, \ldots, \alpha_{v} \in \Delta$. Then, using Lemma 3.1, we obtain

$$
(\Theta(\psi, f) \mid T)(z)=\sum_{i=1}^{\nu} c_{i}\left(\Theta(\psi, f) \mid \alpha_{i}\right)(z)=\Theta\left(\sum_{i=1}^{\nu} c_{i} \psi^{\alpha_{i}}, f\right)(z) .
$$

Since $\psi^{\alpha_{i}}=\psi_{\infty}\left[\pi_{p}\left(\rho\left(\alpha_{i}^{-1}\right)\right) \psi_{p}\right] \prod_{p^{\prime} \neq p} \psi_{p^{\prime}}$, we have

$$
\sum_{i=1}^{\nu} c_{i} \psi^{\alpha_{i}}=\psi_{\infty}\left[\sum_{i=1}^{\nu} c_{i} \pi_{p}\left(\rho\left(\alpha_{i}^{-1}\right)\right) \psi_{p}\right] \prod_{p^{\prime} \neq p} \psi_{p^{\prime}}
$$

On the other hand, we have

$$
\varepsilon \circ \widetilde{\rho}(T)=\varepsilon\left(\sum_{i=1}^{\nu} c_{i}\left(\Gamma_{0}^{n}(q) \rho\left(\alpha_{i}\right)\right)\right)=\sum_{i=1}^{\nu} c_{i}\left(\Gamma_{p}^{n}(q) \rho\left(\alpha_{i}\right)\right)=\sum_{i=1}^{\nu} c_{i}\left(\rho\left(\alpha_{i}^{-1}\right) \Gamma_{p}^{n}(q)\right) .
$$

Using the formula for the action of $H\left(\Gamma_{p}^{n}(q), \Delta_{p}^{n}(q)\right)$ on $\psi_{p}$, we obtain

$$
\pi_{p}(\varepsilon \circ \tilde{\rho}(T)) \psi_{p}=\sum_{i=1}^{\nu} c_{i}\left(\pi_{p}\left(\rho\left(\alpha_{i}^{-1}\right)\right) \psi_{p} .\right.
$$

Hence we have

$$
\sum_{i=1}^{\nu} c_{i} \psi^{\alpha_{i}}=\psi_{\infty}\left[\tilde{\pi}_{p}^{\prime}(\varepsilon \circ \widetilde{\rho}(T)) \psi_{p}\right] \prod_{p^{\prime} \neq p} \psi_{p^{\prime}},
$$

and therefore the proposition follows.

Let $T^{\prime}=\sum_{i=1}^{v^{\prime}} c_{i}^{\prime}\left(U_{p} \alpha_{i}^{\prime}\right) \in H\left(U_{p}, O_{p}\right)$ with $\alpha_{1}^{\prime}, \ldots, \alpha_{v^{\prime}}^{\prime} \in O_{p}$. We assume that the elements $\alpha_{i}$ are chosen in such a way that we also have $T^{\prime}=\sum_{i=1}^{v^{\prime}} c_{i}^{\prime}\left(\alpha_{i}^{\prime} U_{p}\right)$; this can be done for double cosets of the form $U_{p} \alpha U_{p}$, and therefore can be done in general. Define the action of $H\left(U_{p}, O_{p}\right)$ on $\psi_{p}$ by

$$
\pi_{p}^{\prime}\left(T^{\prime}\right) \psi_{p}=\sum_{i=1}^{v^{\prime}} c_{i}^{\prime} \pi_{p}^{\prime}\left(\alpha_{i}^{\prime}\right) \psi_{p}
$$

PROPOSITION 3.3. For each $T^{\prime} \in H\left(U_{p}, O_{p}\right)$ we have

$$
\Theta\left(\psi, f \mid T^{\prime}\right)(z)=\Theta\left(\psi^{\prime}, f\right)(z),
$$

where

$$
\psi^{\prime}=\prod_{v} \psi_{v}^{\prime}, \quad \psi_{v}^{\prime}= \begin{cases}\psi_{v} & \text { for } v \neq p \\ \pi_{p}^{\prime}\left(T^{\prime}\right) \psi_{p} & \text { for } v=p\end{cases}
$$


PROOF. Let $T^{\prime}=\sum_{i=1}^{v^{\prime}} c_{i}^{\prime}\left(U_{p} \alpha_{i}^{\prime}\right) \in H\left(U_{p}, O_{p}\right)$ with the elements $\alpha_{1}^{\prime}, \ldots, \alpha_{v^{\prime}}^{\prime} \in O_{p}$ chosen such that $T^{\prime}=\sum_{i=1}^{\nu^{\prime}} c_{i}^{\prime}\left(\alpha_{i}^{\prime} U_{p}\right)$ as above. Then for each $i$ we have

$$
\begin{aligned}
\int_{O_{\mathbf{Q}} \backslash O_{A}} & \left\langle\sum_{X \in V^{n}} \pi(\rho(g)) \pi^{\prime}\left(g^{\prime}\right) \psi(X), f\left(g^{\prime} \alpha_{i}^{\prime-1}\right)\right\rangle d g^{\prime} \\
= & \int_{O_{\mathbf{Q}} \backslash O_{\Lambda}}\left\langle\sum_{X \in V^{n}} \pi(\rho(g)) \pi^{\prime}\left(g^{\prime}\right)\left(\pi^{\prime}\left(\alpha_{i}^{\prime}\right) \psi\right)(X), f\left(g^{\prime}\right)\right\rangle d g^{\prime}
\end{aligned}
$$

for all $g \in \mathbb{G}(\mathbb{A})$, since the measure $d g^{\prime}$ is right invariant. Thus we obtain

$$
\begin{aligned}
\Theta\left(\psi, f \mid T^{\prime}\right)(z)= & \left(\operatorname{det}^{m / 2} \otimes \mu\right)\left(J\left(\rho\left(\delta g_{\infty}\right), i I_{n}\right)\right) \\
& \times \int_{O_{Q} \backslash O_{A}}\left\langle\sum_{X \in V^{n}} \pi(\rho(g)) \pi^{\prime}\left(g^{\prime}\right)\left(\sum_{i=1}^{v^{\prime}} c_{i}^{\prime} \pi^{\prime}\left(\alpha_{i}^{\prime}\right) \psi\right)(X), f\left(g^{\prime}\right)\right) d g^{\prime} \\
= & \Theta\left(\psi^{\prime}, f\right)(z)
\end{aligned}
$$

with $\psi^{\prime}=\sum_{i=1}^{\nu^{\prime}} c_{i}^{\prime} \pi^{\prime}\left(\alpha_{i}^{\prime}\right) \psi$. However, since $\alpha_{i}^{\prime} \in O_{p}$ for each $i$, we have

$$
\sum_{i=1}^{v^{\prime}} c_{i}^{\prime} \pi^{\prime}\left(\alpha_{i}^{\prime}\right) \psi=\prod_{v} \psi_{v}^{\prime}
$$

with

$$
\psi_{v}^{\prime}= \begin{cases}\psi_{v} & \text { for } v \neq p \\ \sum_{i=1}^{v^{\prime}} c_{i}^{\prime} \pi^{\prime}\left(\alpha_{i}^{\prime}\right) \psi_{p} & \text { for } v=p .\end{cases}
$$

Hence the proof of the proposition is complete.

LEMMA 3.4. Let $\varpi$ be the dimension of a maximal isotropic subgroup of $V_{p}$. If $p \nmid 2 q$, then there are surjective homomorphisms

$$
\eta_{m, n}: H\left(U_{p}, O_{p}\right) \rightarrow H\left(\Gamma_{p}^{n}(q), \Delta_{p}^{n}(q)\right)
$$

for $n \leq \varpi$ and

$$
\eta_{n, m}: H\left(\Gamma_{p}^{n}(q), \Delta_{p}^{n}(q)\right) \rightarrow H\left(U_{p}, O_{p}\right)
$$

for $n \geq \varpi$ such that

$$
\pi_{p}^{\prime}\left(T^{\prime}\right) \psi_{p}=\pi_{p}\left(\eta_{m, n}\left(T^{\prime}\right)\right) \psi_{p}, \quad \pi_{p}\left(T^{\prime \prime}\right) \psi_{p}=\pi_{p}^{\prime}\left(\eta_{n, m}\left(T^{\prime \prime}\right)\right) \psi_{p}
$$

for all $T^{\prime \prime} \in H\left(\Gamma_{p}^{n}(q), \Delta_{p}^{n}(q)\right)$ and $T^{\prime} \in H\left(U_{p}, O_{p}\right)$. 
ProOF. See [8, Remark 4.4].

THEOREM 3.5. Let $p$ be a prime with $p \nmid 2 q$, and consider the Hecke operators $T \in H(\Gamma, \Delta)$ and $T^{\prime} \in H\left(U_{p}, O_{p}\right)$ given as follows:

(i) If $n \geq \varpi$, let $T$ be an arbitrary element of $H(\Gamma, \Delta)$, and set $T^{\prime}=\eta_{n, m}(\varepsilon \circ$ $\tilde{\rho}(T))$.

(ii) If $n \leq \varpi$, let $T \in H(\Gamma, \Delta)$ and $T^{\prime} \in H\left(U_{p}, O_{p}\right)$ be elements satisfying the condition $\varepsilon \circ \widetilde{\rho}(T)=\eta_{m, n}\left(T^{\prime}\right)$.

Then the Hecke operators $T$ and $T^{\prime}$ satisfy the relation

$$
(\Theta(\psi, f) \mid T)(z)=\Theta\left(\psi, f \mid T^{\prime}\right)(z)
$$

for $z \in \mathscr{D}, \psi \in \mathscr{S}\left(V_{\AA}^{n}\right)$ and $f \in \mathscr{A}_{\lambda}\left(O_{\mathbb{Q}} \backslash O_{\AA} / U\right)$.

Proof. Let $T$ be an element of $H(\Gamma, \Delta)$. Then by Proposition 3.2, we have

$$
(\Theta(\psi, f) \mid T)(z)=\Theta\left(\psi^{0}, f\right)(z)
$$

for all $z \in \mathscr{D}$, where

$$
\psi^{0}=\prod_{v} \psi_{v}^{0}, \quad \psi_{v}^{0}= \begin{cases}\psi_{v} & \text { for } v \neq p \\ \pi_{p}(\varepsilon \circ \widetilde{\rho}(T)) \psi_{p} & \text { for } v=p .\end{cases}
$$

We first assume that $n \geq \varpi$, and let $T^{\mathcal{L}}=\eta_{n, m}(\varepsilon \circ \widetilde{\rho}(T))$. Then by Proposition 3.3 we have

$$
\Theta\left(\psi, f \mid T^{\prime}\right)(z)=\Theta\left(\psi^{\prime}, f\right)(z)
$$

where

$$
\psi^{\prime}=\prod_{v} \psi_{v}^{\prime}, \quad \psi_{v}^{\prime}= \begin{cases}\psi_{v} & \text { for } v \neq p \\ \pi_{p}^{\prime}\left(\eta_{n, m}(\varepsilon \circ \widetilde{\rho}(T))\right) \psi_{p} & \text { for } v=p\end{cases}
$$

However, using Lemma 3.4, we get

$$
\pi_{p}^{\prime}\left(\eta_{n, m}(\varepsilon \circ \widetilde{\rho}(T))\right) \psi_{p}=\pi_{p}(\varepsilon \circ \widetilde{\rho}(T)) \psi_{p} .
$$

Hence we obtain the relation $\Theta(\psi, f) \mid T=\Theta\left(\psi, f \mid T^{\prime}\right)$ for the case of (i). As for the case of (ii), we assume that there is an element $T^{\prime} \in H\left(U_{p}, O_{p}\right)$ satisfying the relation $\varepsilon \circ \rho(T)=\eta_{m, n}\left(T^{\prime}\right)$. By Lemma 3.4 we obtain

$$
\pi_{p}(\varepsilon \circ \widetilde{\rho}(T)) \psi_{p}=\pi_{p}\left(\eta_{m, n}\left(T^{\prime}\right)\right) \psi_{p}=\pi_{p}^{\prime}\left(T^{\prime}\right) \psi_{p} .
$$

Using this and Proposition 3.3, we obtain the desired relation in this case, and therefore the proof of the theorem is complete. 


\section{Spin groups}

In this section we apply the results of the previous sections to the case of spin groups associated to Clifford algebras. First, we review the construction of spin groups (see for example [10, Appendix] for details). Let $W$ be a real vector space of dimension $v$ defined over $\mathbb{Q}$, and let $S$ be a nondegenerate symmetric bilinear form of signature $(p, q)$ for some positive integer $p$ defined over $\mathbb{Q}$. Let $\mathscr{T}(W)=\bigoplus_{r=0}^{\infty} W^{\otimes r}$ be the tensor algebra of $W$, and let $\mathscr{I}_{S}$ be the two-sided ideal of $\mathscr{T}(W)$ generated by the set

$$
\{x \otimes x-S(x, x) \mid x \in W\} .
$$

Then the Clifford algebra of $(W, S)$ is given by

$$
\mathscr{C}=\mathscr{C}(V, S)=\mathscr{T}(W) / \mathscr{I}_{S}
$$

Let $\left\{e_{1}, \ldots, e_{\nu}\right\}$ with $v=p+q$ be an orthogonal basis of $V$ such that

$$
S\left(e_{i}, e_{j}\right)=\delta_{i j} \alpha_{i}
$$

for some $\alpha_{1}, \ldots, \alpha_{\nu} \in \mathbb{R}$, where $\delta_{i j}$ is the Kronecker delta. If $W$ is identified with its image in $\mathscr{C}$, then it is known that $\operatorname{dim} \mathscr{C}=2^{v}$ and that the set

$$
\{1\} \cup\left\{e_{i_{1}} \cdots e_{i_{v}} \mid 1 \leq i_{1}<\cdots<i_{r} \leq \nu, 1 \leq r \leq v\right\}
$$

is a basis of $\mathscr{C}$. Thus $\mathscr{C}$ is an associative algebra generated by $e_{1}, \ldots, e_{\nu}$ satisfying the conditions

$$
e_{i}^{2}=\alpha_{i}, \quad e_{i} e_{j}+e_{j} e_{i}=0
$$

for $1 \leq i, j \leq v$ with $i \neq j$. We set

$$
\begin{aligned}
& \left.\mathscr{C}^{+}=\left\langle e_{i_{1}} \cdots e_{i_{v}}\right| 1 \leq i_{1}<\cdots<i_{r} \leq v, r \text { even }\right\rangle_{\mathbb{R}}, \\
& \left.\mathscr{C}^{-}=\left\langle e_{i_{1}} \cdots e_{i_{v}}\right| 1 \leq i_{1}<\cdots<i_{r} \leq \nu, r \text { odd }\right\rangle_{\mathbf{R}} .
\end{aligned}
$$

Then $\mathscr{C}^{+}$is a subalgebra of $\mathscr{C}$ of dimension $2^{\nu-1}$, and we have

$$
\mathscr{C}=\mathscr{C}^{+} \oplus \mathscr{C}^{-}, \quad\left(\mathscr{C}^{+}\right)^{2}=\left(\mathscr{C}^{-}\right)^{2}=\mathscr{C}^{+}, \quad \mathscr{C}^{+} \mathscr{C}^{-}=\mathscr{C}^{-} \mathscr{C}^{+}=\mathscr{C}^{-} .
$$

Let $\iota$ be the canonical involution of $\mathscr{C}$ defined by $e_{i}^{t}=e_{i}$ for $1 \leq i \leq n$. Then the spin group is given by

$$
\operatorname{Spin}(W, S)=\left\{g \in \mathscr{C}^{+} \mid g^{t} g=1, g W g^{-1}=W\right\} .
$$


Given $\operatorname{Spin}(W, S)$, we set

$$
\phi(g) x=g x g^{-1}
$$

for all $x \in W$. Then we have $\phi(g) \in S O(W, S)$, and the map

$$
\phi: \operatorname{Spin}(W, S) \rightarrow S O(W, S)
$$

is a two-fold covering of $S O(W, S)$.

Let $a$ be an element $\mathscr{C}^{+}$with $a^{l}=-a$, and let $b_{1}$ and $b_{2}$ be elements of $\mathscr{C}^{+}$and $\mathscr{C}^{ \pm}$, respectively, such that

$$
b_{1}^{2}+(-1)^{q(q+1) / 2} b_{2}^{2}=-1, \quad b_{1} b_{2}+b_{2} b_{1}=0,
$$

and the bilinear map

$$
(x, y) \mapsto \operatorname{tr}\left(b_{1} a x^{\imath} y\right)+\operatorname{tr}\left(b_{2} a x^{\imath} e_{-} y\right)
$$

for $x, y \in \mathscr{C}^{+}$is symmetric and positive definite. We set

$$
A(x, y)=\operatorname{tr}\left(a x^{c} y\right), \quad I(x)=x b_{1}+e_{-} x b_{2} .
$$

for all $x, y \in \mathscr{C}^{+}$, where $e_{-}=e_{p+1} \cdots e_{\nu}$.

PROPOSITION 4.1. Let $A$ and $I$ be as in (4.1). Then $A$ is a nondegenerate alternating bilinear form on $\mathscr{C}^{+}$, and I is a complex structure on $\mathscr{C}^{+}$such that $(x, y) \mapsto A(x, I y)$ is symmetric and positive definite and

$$
A(g x, g y)=A(x, y), \quad I(g x)=g I(x)
$$

for all $g \in \operatorname{Spin}(W, S)$ and $x, y \in \mathscr{C}^{+}$.

ProOF. See [9, Section 2].

By Proposition 4.1 we see that the left multiplication map

$$
\widetilde{\rho}(g): x \mapsto g x
$$

determines a homomorphism

$$
\widetilde{\rho}: \operatorname{Spin}(W, S) \rightarrow \operatorname{Sp}\left(\mathscr{C}^{+}, A\right)
$$

Now we assume that $p=2$, that is the signature of $S$ is $(2, q)$. Then it is known (see [9]) that the symmetric space $\mathscr{D}=G / K$ associated to a maximal compact subgroup of the spin group $G=\operatorname{Spin}(W, S)$ has a $G$-invariant complex structure. We choose 
a basis of $\mathscr{C}^{+}$in such a way that $S p\left(\mathscr{C}^{+}, A\right)$ can be identified with $\operatorname{Sp}\left(2^{\nu}, \mathbb{R}\right)$ with $v=2+q$ and denote by

$$
\rho: \operatorname{Spin}(W, S) \rightarrow \operatorname{Sp}\left(2^{\nu}, \mathbb{R}\right)
$$

the homomorphism induced by $\tilde{\rho}$. Let $\tau: \mathscr{D} \rightarrow \mathscr{H}_{2 v}$ be a holomorphic map that is equivariant with respect to $\rho$, and choose an arithmetic subgroup $\Gamma$ of $G$ as in Section 2. Applying Theorem 2.6 and Theorem 3.5, we obtain the following result.

THEOREM 4.2. There exist theta series liftings from automorphic forms on orthogonal groups to automorphic forms on spin groups of type $(2, q)$ and such liftings are compatible with appropriate Hecke operator actions.

\section{References}

[1] R. Howe, $\theta$-series and invariant theory, Proc. Sympos. Pure Math. 33, Part 1 (Amer. Math. Soc., Providence, 1979) pp. 275-286.

[2] M. Kashiwara and M. Vergne, 'On the Segal-Shale-Weil representations and harmonic polynomials', Invent. Math. 44 (1978), 1-47.

[3] M. Kuga, Fiber varieties over a symmetric space whose fibers are abelian varieties I, II (Univ. of Chicago, Chicago, 1963/64).

[4] M. H. Lee, 'Conjugates of equivariant holomorphic maps of symmetric domains', Pacific J. Math. 149 (1991), 127-144.

[5] —_. 'Mixed Siegel modular forms and Kuga fiber varieties', Illinois J. Math. 38 (1994), 692700.

[6] — ' 'Mixed automorphic forms on semisimple Lie groups', Ilinois J. Math. 40 (1996), 464478.

[7] _ - 'Mixed automorphic vector bundles on Shimura varieties', Pacific J. Math. 173 (1996), $105-126$.

[8] S. Rallis, 'Langlands' functoriality and the Weil representation', Amer. J. Math. 104 (1982), 469515.

[9] I. Satake, 'Clifford algebras and families of Abelian varieties', Nagoya Math. J. 27 (1966), 435446.

[10] - Algebraic structures of symmetric domains (Princeton Univ. Press, Princeton, 1980).

[11] V. Zhuravlev, 'Spherical theta-series and Hecke operators', Proc. Steklov Inst. Math. (1995), 87110.

Department of Mathematics

University of Northern Iowa

Cedar Falls, IA 50614

USA

e-mail: lee@math.uni.edu 